\title{
Impactos toxicológicos de herbicidas recomendados para a cultura do milho em ninfas do predador Podisus nigrispinus (Hemiptera: Pentatomidae) ${ }^{1}$
}

\author{
Toxicological impact of herbicides recommended for corn crop in nymphs of \\ Podisus nigrispinus (Hemiptera: Pentatomidae) predator
}

\author{
Silma da Silva Camilo² ${ }^{2}$ Marcus Alvarenga Soares ${ }^{3}$; José Barbosa dos Santos ${ }^{3}$; Sebastião \\ Lourenço de Assis Júnior ${ }^{4}$; Evander Alves Ferreira ${ }^{5}$; Claubert Wagner Guimarães de Menezes ${ }^{6}$
}

Resumo - A presença de plantas daninhas na cultura do milho (Zea mays L.) pode ocasionar altas perdas na produção. O controle químico das plantas daninhas é o mais utilizado nesta cultura, mas pode afetar negativamente os inimigos naturais presentes nas lavouras. O objetivo do trabalho foi avaliar a toxicidade de herbicidas registrados para a cultura do milho a ninfas do predador Podisus nigrispinus Dallas, 1851 (Hemiptera: Pentatomidae). Avaliou-se o efeito de atrazine, nicosulfuron, mesotrione, paraquat e o controle à base de água destilada, sobre três estádios imaturos do inseto (terceiro, quarto e quinto), em doses equivalentes à metade, ao dobro, a quatro e dez vezes à recomendada para a cultura do milho. Os herbicidas foram aplicados sobre potes plásticos de $500 \mathrm{~mL}$ contendo, cinco ninfas de cada estádio do inseto, constituindo a unidade amostral, com seis repetições. Após 96 horas foi realizada a contagem dos sobreviventes. Para todos os estádios de $P$. nigrispinus o aumento das doses dos herbicidas ocasionou decréscimo na taxa de sobrevivência. Esta foi menor para os insetos expostos ao atrazine e paraquat. $\mathrm{O}$ mesotrione foi o menos tóxico e o nicosulfuron apresentou toxicidade mediana. Conclui-se que as ninfas do terceiro ao quinto estádio do inimigo natural $P$. nigrispinus são mais suscetíveis aos herbicidas atrazine e paraquat. Além disso, mesotrione e nicosulfuron podem ser utilizados na cultura do milho, visando preservar a população deste predador.

Palavras-chaves: controle biológico, plantas daninhas, sobrevivência, percevejo

Abstract - Weeds presence in corn crop (Zea mays L.) may cause high losses in production. Chemical control of these plants is the most used, but may adversely affect natural enemies present in crops. Therefore, the objective of this study was to evaluate herbicides toxicity $\mathrm{s}$ registered for corn crop to nymphs of Podisus nigrispinus Dallas, 1851 (Heteroptera:

\footnotetext{
${ }^{1}$ Recebido para publicação em 21/09/2012 e aceito em 28/12/2012.

${ }^{2}$ Mestranda do Programa de Pós-Graduação em Produção Vegetal, Universidade Federal dos Vales do Jequitinhonha e Mucuri, Rodovia MGT 367, Km 583, nº 5000, CEP: 39100-000, Alto da Jacuba, Diamantina - MG, Brasil. E-mail: <dsilma@yahoo.com.br>. (Autor para correspondência);

${ }^{3}$ Docentes do Programa de Pós-Graduação em Produção Vegetal, Universidade Federal dos Vales do Jequitinhonha e Mucuri;

${ }^{4}$ Docente do Programa de Pós-Graduação em Ciência Florestal, Universidade Federal dos Vales do Jequitinhonha e Mucuri;

${ }^{5}$ Pós-doutorando do Programa de Pós-Graduação em Produção Vegetal, Universidade Federal dos Vales do Jequitinhonha e Mucuri;

${ }^{6}$ Doutorando do Programa de Pós-Graduação em Agronomia/Fitotecnia, Universidade Federal de Lavras, Campus Universitário, Cx Postal: 3037 37200-000, CEP: 37200-000, Lavras - MG, Brasil.
} 
Pentatomidae) predator. It was evaluated the effect of atrazine, nicosulfuron, mesotrione, paraquat and its control based on distilled water over three immature stages of insects (third, fourth and fifth) in doses equivalent to half, to twice, four and ten times the recommended one for corn crop. Herbicides were applied over plastic pots of $500 \mathrm{~mL}$ each, containing five nymphs in each stage of the insect, constituting the experimental plot, with six replications. After 96 hours was performed the counting of survivors individuals. For all stages of $P$. nigrispinus increasing herbicides doses caused a decrease in survival rate. This one was inferior for insects exposed to atrazine and paraquat herbicides. Mesotrione herbicide was the least toxic and nicosulfuron showed median toxicity. It was concluded that nymphs from third to fifth stage of $P$. nigrispinus natural enemy are more susceptible to atrazine and paraquat herbicides. Moreover, mesotrione and nicosulfuron may be used for controlling weeds in corn crop when the objective is to preserve the population of this predator.

Keywords: biological control, weed, survival, bug

\section{Introdução}

O milho (Zea mays) é uma cultura importante no país para a alimentação humana e animal (Carvalho et al., 2007). A área cultivada com este cereal na safra de 2010/2011 foi de 13,8 milhões de hectares, com produção de cerca de 57,4 milhões de toneladas de grãos (CONAB, 2012).

Um dos fatores que afetam o rendimento desta cultura é a interferência exercida pelas plantas daninhas, que podem provocar redução na produtividade de grãos (Timossi et al., 2011). Dentre os métodos de controle disponíveis, o químico tem se destacado pela eficiência, rapidez na operação e economia nos custos (Jakelaitis et al., 2005). No entanto, a contaminação ambiental provocada pelo uso de agrotóxicos tem gerado preocupações relacionadas ao emprego inadequado desses compostos (Costa et al., 2007). Por isso, é necessário melhorar a eficiência nas aplicações em lavouras, diminuindo desperdícios, aumentando o retorno econômico e evitando contaminações (Reynaldo et al., 2011).

No Brasil, a lagarta-do-cartucho, Spodoptera frugiperda (Lepidoptera: Noctuidae) é a principal praga da cultura do milho (Mendes et al., 2011). As plantas são suscetíveis ao ataque deste inseto em todos os estádios do seu ciclo de desenvolvimento, com perdas severas de rendimento devido à redução da área fotossintética, ocasionada pela destruição de folhas e da região do cartucho (Lima et al., 2010). O percevejo predador, Podisus nigrispinus (Hemiptera: Pentatomidae), tem ocorrência natural em inúmeros agroecossistemas, sendo uma das espécies mais comuns da subfamília Asopinae no Brasil (Torres et al., 2006). É um importante inimigo natural de muitas pragas em culturas de relevância econômica (Medeiros et al., 2003), incluindo lagartas de S. frugiperda (Oliveira et al., 2004).

O controle de plantas daninhas influencia todo o agroecossistema, afetando artrópodes herbívoros e seus inimigos naturais (Albajes et al., 2009), principalmente, em função do método químico, que é o mais utilizado. Assim, a aplicação de herbicidas pode provocar efeitos deletérios a estes organismos.

Ninfas do terceiro, quarto e quinto estádios de $P$. nigrispinus são muito móveis no campo, se alimentando de forma intensiva para completar seu desenvolvimento, sendo assim, as fases mais suscetíveis desta espécie ao contato direto com agrotóxicos. Ninfas do primeiro e segundo estádios praticamente não se alimentam e são pouco móveis (Silva et al., 2009). Já a fase adulta, apesar da maior mobilidade, é também menos afetada pela exposição a estes compostos, possivelmente 
por possuir mecanismos eficientes de Material e Métodos

destoxificação (Evangelista Jr. et al., 2002).

No entanto, poucos trabalhos foram realizados visando analisar o efeito dos herbicidas em inimigos naturais presentes nos cultivos agrícolas. Alguns estudos demonstram que herbicidas registrados para a cultura do milho podem afetar o parasitoide Trichogramma pretiosum (Hymenoptera: Trichogrammatidae), sendo Callisto ${ }^{\circledR}$, Equip Plus $^{\circledR}$, Extrazin SC ${ }^{\circledR}$, Primóleo ${ }^{\circledR}$, Provence 750 $\mathrm{WG}^{\circledR}$ e Siptran $500 \mathrm{SC}^{\circledR}$ considerados inócuos; Agrisato $480 \mathrm{SL}^{\circledR}$, Gesaprim GrDA ${ }^{\circledR}$, Glifos ${ }^{\circledR}$, Glyphosate Nortox ${ }^{\circledR}$, Gliz 480 SL $^{\circledR}$, Polaris $^{\circledR}$, Primatop SC ${ }^{\circledR}$, Sanson 40 SC $^{\circledR}$, Trop ${ }^{\circledR}$ e Zapp $\mathrm{Qi}^{\circledR}$, levemente nocivos; Finale ${ }^{\circledR}$, Herbadox ${ }^{\circledR}$, Poast $^{\circledR}, \quad$ Roundup Original $^{\circledR}$, Roundup Transorb $^{\circledR}$ e Roundup $\mathrm{WG}^{\circledR}$, moderadamente nocivos; e Gramoxone $200^{\circledR}$ e Primestra Gold ${ }^{\circledR}$ nocivos (Stefanello Júnior et al., 2008).

Além disso, segundo Soares et al. (1995) os herbicidas MSMA (ácido monosódico metil arsonato) e fluazyfop-p-butil provocaram redução populacional do predador Cycloneda sanguinea (Coleoptera: Coccinellidae) e MSMA de Doru lineare (Dermaptera: Forficulidae), aos sete dias após a aplicação na cultura do algodoeiro.

De acordo com Manzoni et al. (2006) herbicidas recomendados para macieira provocaram reduções no parasitismo de $T$. pretiosum, em relação à testemunha (água), sendo Polaris ${ }^{\circledR}$ classificado como levemente nocivo; Finale ${ }^{\circledR}$ e Roundup Original ${ }^{\circledR}$ como moderadamente nocivos. Já os herbicidas amônio-glufosinato e oxyfluorfen, utilizados para a cultura do eucalipto, reduziram o parasitismo e a emergência de Palmistichus elaeisis (Hymenoptera: Eulophidae) (Menezes et al., 2012).

Portanto, o trabalho teve como objetivo avaliar a toxicidade dos herbicidas atrazine, nicosulfuron, mesotrione e paraquat em ninfas de terceiro, quarto e quinto estádios do predador $P$. nigrispinus.
$\mathrm{O}$ experimento foi conduzido no Laboratório de Entomologia da Universidade Federal dos Vales do Jequitinhonha e Mucuri, em Diamantina, estado de Minas Gerais, a $25 \pm$ $2{ }^{\circ} \mathrm{C}, 70 \pm 10 \%$ de umidade relativa e fotofase de 12 horas.

Os testes de toxicidade foram realizados utilizando-se ninfas do terceiro, quarto e quinto estádios de desenvolvimento de $P$. nigrispinus, provenientes da criação massal do laboratório. Estas ninfas foram criadas em potes de 1000 $\mathrm{mL}$, alimentadas com pupas de Tenebrio molitor (Coleoptera: Tenebrionidae) e água destilada disponibilizada em tubos de vidro (tipo anestésico odontológico) com a extremidade coberta por um chumaço de algodão.

Avaliou-se o efeito dos herbicidas atrazine, nicosulfuron, mesotrione, paraquat e o controle à base de água destilada sobre os três estádios do inseto, em doses equivalentes à metade, ao dobro, a quatro e a dez vezes à dose recomendada para a cultura do milho (MAPA, 2010). As doses utilizadas em $\mathrm{kg}$ de i.a. (ingrediente ativo) por hectare, foram: 1,10; 4,$40 ; 8,80$ e 22,00 para atrazine, 0,$03 ; 0,11$; 0,22 e 0,55 para nicosulfuron, 0,$08 ; 0,34 ; 0,67$ e 1,68 para mesotrione e 0,$22 ; 0,90 ; 1,80$ e 4,50 para paraquat.

Aspergiu-se $0,2 \mathrm{~mL}$ da solução herbicida, equivalente a uma taxa de aplicação de $200 \mathrm{~L} \mathrm{ha}^{-1}$, sobre cinco ninfas de $P$. nigrispinus de cada estádio dentro de potes de plástico de $500 \mathrm{~mL}$, com área basal de 70,9 $\mathrm{cm}^{2}$. Para que a aplicação do herbicida fosse realizada da maneira uniforme, de forma a cobrir todo o fundo do recipiente, utilizou-se uma seringa com a extremidade da agulha adaptada para pulverização, em forma de leque, de acordo com metodologia de Souza et al. (2000).

Cada recipiente constituiu uma unidade amostral com seis repetições, em delineamento inteiramente casualizado. Após 96 horas, o número de sobreviventes em cada recipiente foi 
contado. Durante este intervalo, os insetos foram alimentados com pupas de T. molitor e água destilada.

Os dados foram submetidos à análise de variância (ANOVA) e quando significativos comparados pelo teste $\mathrm{t}$ a $10 \%$ de significância. Foram ajustadas curvas de regressão para a taxa de sobrevivência das ninfas de $P$. nigrispinus.

\section{Resultados e Discussão}

O aumento nas doses dos herbicidas atrazine, nicosulfuron, mesotrione e paraquat ocasionou decréscimo na taxa de sobrevivência de todos os estádios de P. nigrispinus (Figura $1)$.

A toxicidade foi diferenciada para cada um dos herbicidas empregados no teste. Dosagens de herbicidas recomendadas pelo fabricante são frequentemente aplicadas em quantidade maior que a necessária, para garantir o controle eficiente das plantas daninhas em diversas condições ambientais e de manejo e, em especial, por causa de falhas na tecnologia de aplicação (Costa et al., 2007).

O herbicida atrazine foi mais tóxico na dose correspondente a dez vezes à recomendada para a cultura do milho $(22 \mathrm{~kg}$ i.a. ha $\left.^{-1}\right)$. Nesta dosagem, ocasionou sobrevivência média de 40,0; 46,7 e 33,3\% para o terceiro, quarto e quinto estádio de $P$. nigrispinus, respectivamente. A exposição às doses equivalentes ao dobro e a quatro vezes à comercial foi mais tóxica para o quarto e quinto estádios. Já os insetos do terceiro estádio apresentaram sobrevivência média de $70,0 \%$, quando em contato com cada das mesmas dosagens (Figura $1 \mathrm{~A})$. O atrazine na dose $2250 \mathrm{~mL}$ i.a. ha $^{-1}$ foi nocivo ao artrópode Collembola (Arthropoda: Ellipura), influenciando a abundância desse organismo no solo ao diminuir sua população (Vilma et al., 2007). No entanto, em um trabalho que avaliou a relação entre atividade microbiana e presença de atrazine no solo, foi verificado que a atividade dos microrganismos não foi afetada pela aplicação do herbicida na dosagem de 15 $\mathrm{kg}$ i.a. ha ${ }^{-1}$ (Kleinschmitt et al., 2006), uma dose superior a quatro vezes à recomendada para a cultura do milho $\left(8,8 \mathrm{~kg}\right.$ i.a. ha $\left.{ }^{-1}\right)$.

A exposição ao nicosulfuron não provocou grande redução nas taxas de sobrevivência de $P$. nigrispinus. Estas foram superiores a 70,0\%, mesmo quando as ninfas foram aspergidas com a maior dosagem (Figura 1 B). A baixa toxicidade deste herbicida também foi observada por Massa et al. (2008), que verificaram a não ocorrência de mortalidade de larvas do terceiro estádio de Helicoverpa zea (Lepidoptera: Noctuidae) imersas em solução nicosulfuron na concentração de $22 \mathrm{mg} \mathrm{L}^{-1}$.

Mesotrione foi o menos tóxico para as ninfas de $P$. nigrispinus, permitindo taxas de sobrevivência entre 90,0 e 100,0\% (Figura 1 C). Devido à introdução recente do mesotrione na agricultura, há poucas informações na literatura relativas ao seu risco ecotoxicológico no ambiente e de seus produtos de degradação (Bonnet et al., 2008). No entanto, esse herbicida apresenta risco baixo a moderado para pássaros, mamíferos e organismos aquáticos (Kreutz et al., 2008). Além disso, o mesotrione puro e na formulação comercial Callisto ${ }^{\circledR}$ afetaram comunidades microbianas do solo reduzindo sua atividade, mas os efeitos só foram detectados em doses muito superiores às recomendadas para o controle de plantas daninhas (Crouzet et al., 2010). Stefanello Jr. et al. (2008) caracterizaram o mesotrione $\left(\right.$ Callisto $\left.^{\circledR}\right)$ como inócuo ao inimigo natural $T$. pretiosum, já que a exposição a esse herbicida não provocou redução significativa na capacidade de parasitismo dos insetos fêmeas. A $\mathrm{CL}_{50} 96 \mathrm{~h}$ de mesotrione para alevinos do peixe jundiá, Rhamdia quelen (Teleostei: Pimelodidae), foi superior a $0,5 \mathrm{~g} \mathrm{~L}^{-1}$, que superou a dose recomendada para o controle de plantas daninhas (Kreutz et al., 2008).

A metade, o dobro e quatro vezes a dose recomendada para a cultura do milho do herbicida paraquat provocaram menores taxas 
de sobrevivência a ninfas do terceiro estádio. Já a maior dose foi mais nociva para o quinto estádio, ocasionando taxa de sobrevivência de $36,67 \%$ (Figura 1 D). A menor taxa de sobrevivência das ninfas de $P$. nigrispinus expostas ao paraquat pode ter ocorrido por influência de seu mecanismo de ação. Este herbicida provoca a formação de radicais livres

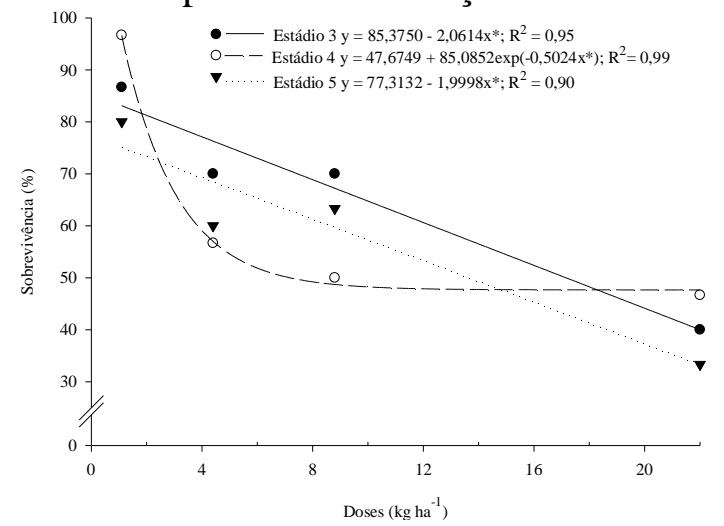

(A)

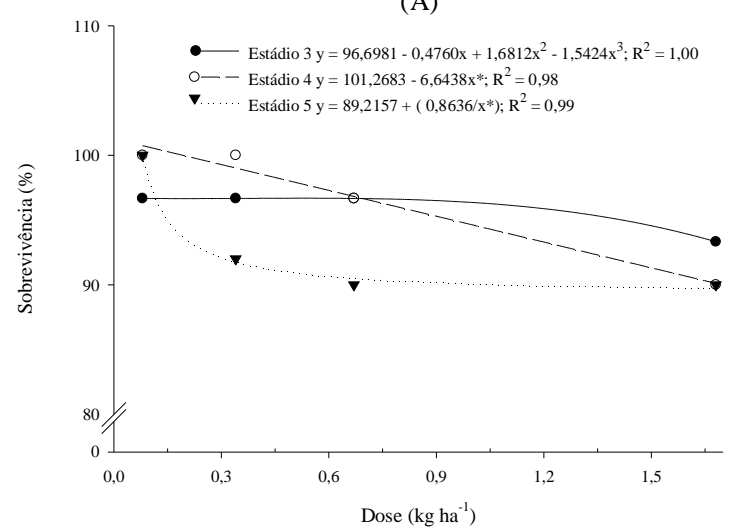

(C) de espécies reativas de oxigênio, como o radical superóxido, o peróxido de hidrogênio e o radical hidroxila, que são instáveis e reagem com ácidos graxos, ocasionando danos às membranas, proteínas e DNA, injúrias aos tecidos (Peter et al., 1992, Almeida et al., 2007).

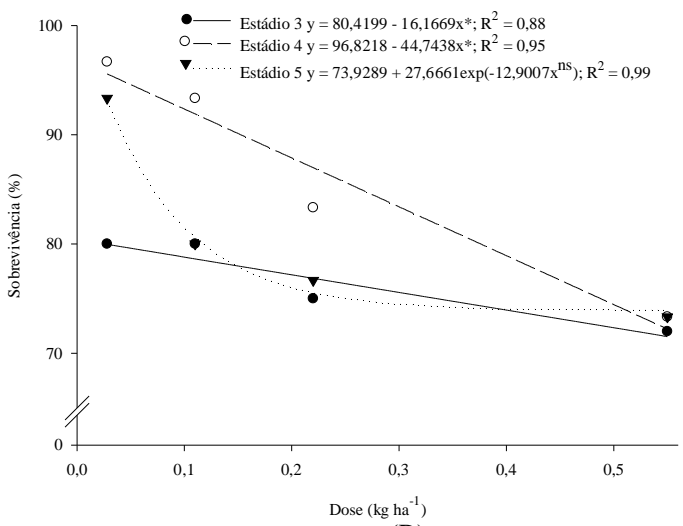

(B)

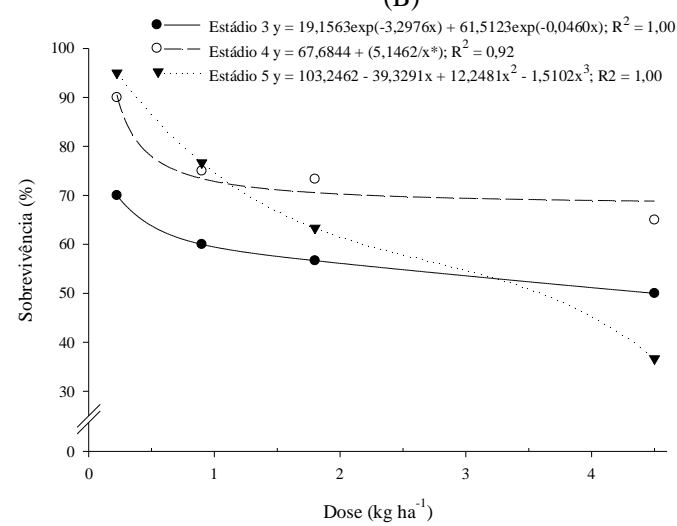

(D)

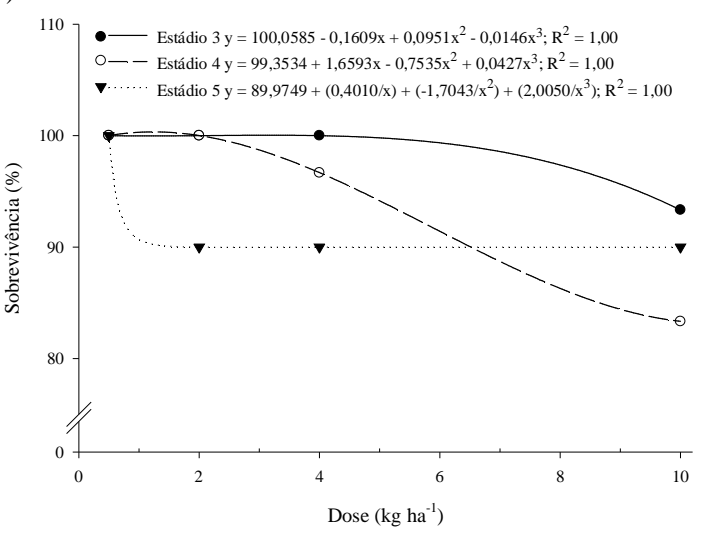

(E)

Figura 1. Curvas de sobrevivência média (\%) de Podisus nigrispinus (Hemiptera: Pentatomidae), 96h após exposição a quatro doses (metade, duas, quatro e dez vezes a dose comercial) dos herbicidas atrazine (A), nicosulfuron (B), mesotrione (C), paraquat (D) e controle à base de água destilada $(\mathrm{E}) . *$ Significativo pelo teste t a $10 \%$ de significância. 
A toxicidade dos herbicidas a ninfas de $P$. nigrispinus pode estar ligada não só aos ingredientes ativos, mas também aos aditivos presentes na formulação comercial, tais como os surfactantes. Além disso, o efeito tóxico pode ser agravado pelo uso de dosagens maiores que as recomendadas para a cultura. Portanto, além da escolha de produtos menos tóxicos a inimigos naturais, é necessário o cuidado na aplicação para se evitar o uso de doses maiores que as recomendadas, comprometendo a eficiência do controle biológico de pragas.

\section{Conclusões}

O aumento das doses de todos os herbicidas testados ocasionou decréscimo na taxa de sobrevivência em todos os estádios de Podisus nigrispinus (Hemiptera: Pentatomidae), sendo a toxicidade diferenciada para cada ingrediente ativo avaliado.

A taxa de sobrevivência do predador foi menor quando expostos a atrazine e paraquat, sendo que o mesotrione foi o menos tóxico e o nicosulfuron apresentou toxicidade mediana.

Produtos à base de atrazine e paraquat podem aumentar a mortalidade de imaturos do predador $P$. nigrispinus no campo, reduzindo sua população e seus benefícios como agentes de controle biológico no agroecossistema.

Mesotrione e nicosulfuron, devido apresentarem baixa e mediana toxicidade ao $P$. nigrispinus permitem alinhar o manejo integrado de pragas e ao de plantas daninhas na cultura do milho.

\section{Agradecimentos}

À Fundação de Amparo à Pesquisa do Estado de Minas Gerais (FAPEMIG) pelo apoio financeiro dado a essa pesquisa.

\section{Referências}

ALBAJES, R.; LUMBIERRES, B.; PONS X. Responsiveness of arthropod herbivores and their natural enemies to modified weed management in corn. Environmental Entomology, v.38, n.3, p.944-954, 2009.

ALMEIDA, G.L. et al. Os riscos e danos nas intoxicações por paraquat em animais domésticos. Ciência Rural, v.37, n.5, p.15061512, 2007.

BONNET, J.L. et al. Toxicity assessment of the herbicides sulcotrione and mesotrione toward two reference environmental microorganisms: Tetrahymena pyriformis and Vibrio fischeri. Archives of Environmental Contamination and Toxicology, v.55, n.4, p.576-583, 2008.

CARVALHO, L.B. et al. Estudo comparativo do acúmulo de massa seca e macronutrientes por plantas de milho var. BR-106 e Brachiaria plantaginea. Planta Daninha, v.25, n.2, p.293301, 2007.

CONAB. Companhia Nacional de Abastecimento. Acompanhamento de safra brasileira: Grãos, Décimo primeiro levantamento, agosto de 2012 / Brasília. Disponível em: http://www.conab.gov.br/OlalaCMS/uploads/ar quivos/12_08_09_10_58_55_boletim_portugue s_agosto_2012.pdf. Acesso em: 10 de agosto de 2012.

COSTA, A.G.F. et al. Efeito da intensidade do vento, da pressão e de pontas de pulverização na deriva de aplicações de herbicidas em préemergência. Planta Daninha, v.25, n.1, p.203210, 2007.

CROUZET, O. et al. Response of soil microbial communities to the herbicide mesotrione: A dose-effect microcosm approach. Soil Biology \& Biochemistry, v.42, n.2, p.193-202, 2010.

EVANGELISTA JR., W.S.; SILVA-TORRES, C.S.A.; TORRES, J.B. Toxicidade de Lufenurom para Podisus nigrispinus (Dallas) (Heteroptera: Pentatomidae). Neotropical Entomology, v.31, n.2, p.319-326, 2002. 
JAKELAITIS, A. et al. Efeitos de herbicidas no consórcio de milho com Brachiaria brizantha. Planta Daninha, v.23, n.1, p.69-78, 2005.

KLEINSCHMITT, A.R.B. et al. Dessorção do herbicida atrazina e atividade microbiana em duas classes de solos do estado do Rio Grande do Sul. Ciência Rural, v.36, n.6, p.1794-1798, 2006.

KREUTZ, L.C. et al. Acute toxicity test of agricultural pesticides on silver catfish (Rhamdia quelen) fingerlings. Ciência Rural, v.38, n.4, p.1050-1055, 2008.

LIMA, M.S. et al. Corn yield response to weed and fall armyworm controls. Planta Daninha, v.28, n.1, p.103-111, 2010.

MANZONI, C.G. et al. Seletividade de agrotóxicos usados na produção integrada de maçã para adultos de Trichogramma pretiosum. Pesquisa Agropecuária Brasileira, v.41, n.10, p.1461-1467, 2006.

MAPA. Ministério da Agricultura Pecuária e Abastecimento. Consulta de Produtos Formulados/2010. Disponível em: http://agrofit.agricultura.gov.br/agrofit_cons/pr incipal_agrofit_cons. Acesso em: 13 jan. 2011.

MASSA, R.; BLEVINS, S.; CHAO, S.L. Role of acetylcholinesterase and glutathione Stransferase following exposure to nicosulfuron and diazinon in Helicoverpa zea. Ecotoxicology and Environmental Safety, v.71, n.71, p.230-235, 2008.

MEDEIROS, R.S. et al. Effect of temperature on life table parameters of Podisus nigrispinus (Het., Pentatomidae) fed with Alabama argillacea (Lep., Noctuidae) larvae. Journal of Applied Entomology, v.127, n.4, p.209-213, 2003.

MENDES, S. M. et al. Respostas da lagarta do cartucho a milho geneticamente modificado expressando a toxina Cry $1 \mathrm{~A}(\mathrm{~b})$. Pesquisa Agropecuária Brasileira, v.46, n.3, p.239244, 2011.
MENEZES, C.W.G. et al. Reproductive and toxicological impacts of herbicides used in Eucalyptus culture in Brazil on the parasitoid Palmistichus elaeisis (Hymenoptera: Eulophidae). Weed Research, v.52, n.6, p.520-525, 2012.

OLIVEIRA, H.N. et al. Desenvolvimento do predador Podisus nigrispinus alimentado com Spodoptera frugiperda e Tenebrio molitor. Pesquisa Agropecuária Brasileira, v.39, n.10, p.947-951, 2004.

PETER, B. et al. Role of lipid peroxidation and DNA damage in paraquat toxicity and the interaction of paraquat with ionizing radiation. Biochemical Pharmacology, v.43, n.4, p.705715, 1992.

REYNALDO, E.F.; MOLIN, J.P. Proposta metodológica para avaliação de controlador automático de seções e pulverização. Engenharia Agrícola, v.31, n.1, p.111-120, 2011.

SILVA, C.A.D. et al. Development and survival of nymphs of Podisus nigrispinus (Heteroptera: Pentatomidae) fed with caterpillars of Chlosyne lacinia saundersii (Lepidoptera: Nymphalidae). Brazilian Archives of Biology and Technology, v.52, n.1, p. 105-109, 2009.

SOARES, J.J.; BRAZ, B.A.; BUSOLI, A.C. Impacto de herbicidas sobre artrópodos benéficos associados ao algodoeiro. Pesquisa Agropecuária Brasileira, v.30, n.9, p.11351140, 1995.

SOUZA, A.P. et al. Lixiviação de glyphosate e imazapyr em solos com diferentes texturas e composição química. I - Método do bioensaio. Planta Daninha, v. 18, n.1, p.5-16, 2000.

STEFANELLO JR., G.J. et al. Seletividade de herbicidas registrados para a cultura do milho a adultos de Trichogramma pretiosum (Hymenoptera: Trichogrammatidae). Planta Daninha, v.26, n.2, p.343-351, 2008. 
TIMOSSI, P.C.; FREITAS, T.T. Eficácia de nicosulfuron isolado e associado com atrazine no manejo de plantas daninhas em milho. Revista Brasileira de Herbicidas, v.10, n.3, p.210-218, 2011.

TORRES, J.B.; ZANUNCIO, J.C.; MOURA, M.A. The predatory stinkbug Podisus nigrispinus: biology, ecology and augmentative releases for lepidopteran larval control in Eucalyptus in Brazil. Biocontrol News and Information, v.27, n.3, p.1-15, 2006.

VILMA, S.L.; SANTOS, H.R.; GONÇALVES, M.C. The effect of the glyphosate, 2,4-D, atrazine e nicosulfuron herbicides upon the edaphic Collembola (Arthropoda: Ellipura) in a no tillage system. Neotropical Entomology, v.36, n.2, p.261-267, 2007. 